REGARDS

SUR LECONOMIE ALLEMANDE

BULLETIN ECONOMIQUE DU CRAC

\section{Regards sur l'économie allemande}

Bulletin économique du CIRAC

$92 \mid 2009$

Varia

\title{
Fiscalité des PME : les effets pervers de la réforme
}

\section{Klaus-Heiner Röhl}

Traducteur : Isabelle Bourgeois

\section{(2) OpenEdition}

Journals

Édition électronique

URL : http://journals.openedition.org/rea/3748

DOI : $10.4000 /$ rea.3748

ISBN : 978-2-8218-0880-5

ISSN : 1965-0787

Éditeur

CIRAC

Édition imprimée

Date de publication : 1 juillet 2009

Pagination : 13-20

ISSN : 1156-8992

\section{Référence électronique}

Klaus-Heiner Röhl, «Fiscalité des PME : les effets pervers de la réforme », Regards sur l'économie

allemande [En ligne], 92 I juillet 2009, mis en ligne le 01 juillet 2011, consulté le 19 avril 2019. URL

http://journals.openedition.org/rea/3748; DOI : 10.4000/rea.3748 


\title{
Fiscalité des PME : les effets pervers de la réforme
}

\author{
Klaus-Heiner Röhl
}

Le principal objectif de la réforme de fond de la fiscalité des entreprises entrée en vigueur le $1^{\text {er }}$ janvier 2008 (Loi Unternehmenssteuerreformgesetz du 14-08-2007) était d'abaisser le taux d'imposition des sociétés de capitaux qui, avec quelque $40 \%$, était très élevé en comparaison internationale. De nombreux Etats avaient réduit la charge fiscale pesant sur leurs entreprises, et le gouvernement allemand devait réagir à cette concurrence accrue entre les sites de production. La réforme visait donc à ramener à $30 \%$ le taux global de prélèvement sur les sociétés de capitaux, sachant qu'en RFA celles-ci sont soumises à deux types d'impôts : l'impôt sur les sociétés (Körperschaftsteuer), uniforme sur l'ensemble du territoire et dont Bund et Länder se partagent le produit à égalité ; et la taxe professionnelle (Gewerbesteuer) qui revient en propre aux communes et dont celles-ci sont libres de fixer les taux.

Or $83 \%$ des entreprises du Mittelstand ne sont pas des sociétés de capitaux, mais des sociétés de personnes dirigées par le propriétaire. Leurs bénéfices sont imposés selon le barème de l'impôt sur le revenu applicable à leur(s) propriétaire(s). S'y ajoute la taxe professionnelle, bien que celle-ci soit déductible de l'IR, et ce, en grande partie dès avant la réforme de 2008. Or en abaissant le barème pour les sociétés de capitaux, on risquait de provoquer une hausse sensible de la charge pesant sur les sociétés de personnes (sauf les TPE). Pour y remédier, la loi de réforme abaissa le barème de l'impôt sur les bénéfices non distribués et améliora les conditions permettant de déduire la taxe professionnelle de l'IR.

Selon les prévisions, ces aménagements se seraient traduits par une baisse de 9 milliards $€$ de l'encours fiscal. Or la Grande coalition avait décidé de plafonner celle-ci à 5 milliards $€$. Diverses mesures élargissant l'assiette des prélèvements et censées générer un apport de 3,8 milliards $€$ furent donc adoptées, dont principalement des règles moins favorables pour les amortissements et l'intégration des baux et intérêts dans le calcul des revenus imposables. L'impact réel de ce nouveau régime sera toutefois plus que délicat à évaluer, la crise mondiale ayant frappé l'économie allemande à la fin 2008; elle comprime les recettes fiscales bien plus drastiquement que l'aurait fait la seule réforme.

Les estimations chiffrées des effets du nouveau régime révèlent néanmoins que l'objectif d'un barème de $30 \%$ n'a pas réellement été atteint. Le taux pesant sur les sociétés de capitaux s'élève actuellement à $31 \%$ en moyenne; la différence provient pour l'essentiel de ce que le gouvernement fédéral a sous-estimé le barème moyen de la taxe professionnelle communale. Bien plus important encore est l'échec de la réforme en ce qui concerne les sociétés de personnes, pour lesquelles le taux s'élève à $37,6 \%$ même lorsqu'elles thésaurisent ou investissent la totalité de leurs bénéfices. La raison principale en est que les bénéfices non distribués sont assimilés fiscalement à des dividendes, donc soumis à l'impôt sur le revenu. Les experts fiscalistes critiquent également les mesures visant à contrefinancer la réforme, en particulier cette " limite d'intérêts » (Zinsschranke) qui réduit la déductibilité des intérêts lors du calcul de l'assiette imposable...

\section{La plupart des entreprises sont des PME au statut de société de personnes}

L'opinion publique ne retient de l'économie que l'image des grands groupes, surtout des sociétés par actions cotées au DAX. Pourtant, l'économie allemande repose en réalité largement sur les PME (le Mittelstand). Selon la définition en

L'économie allemande repose sur le Mittelstand 
Le critère fiscal déterminant n'est pas la taille, mais le statut juridique de l'entreprise vigueur outre-Rhin, appartiennent à cette catégorie les sociétés de moins de 500 salariés ; de moins de 250 selon la définition de l'UE. Mais en ce qui concerne le chiffre d'affaires, les deux définitions le plafonnent en dessous de 50 millions $€$. A cette définition quantitative, il conviendrait d'ajouter un critère qualitatif qui révèle mieux la spécificité du Mittelstand: si dans cette catégorie prédominent les sociétés de personnes, leur capital est le plus souvent détenu par la famille fondatrice, et celle-ci dirige 'son' entreprise.

Si on inclut les TPE dans la catégorie des sociétés moyennes, alors 99,5\% des entreprises recensées en Allemagne appartiennent au Mittelstand, soit plus de 3,5 millions d'entreprises dont plus de 2 millions occupent des salariés en dehors du propriétaire. Le Mittelstand est également prédominant si on considère la population active : il occupe plus de 21 millions de personnes, ce qui représente près des quatre cinquièmes des emplois soumis à cotisations sociales. Par ailleurs, les PME occupent plus de 4 millions de travailleurs indépendants.

Or l'appartenance au Mittelstand n'est pas un critère donnant lieu à un régime fiscal unique. En effet, le critère déterminant pour l'impôt est le statut juridique de l'entreprise : les sociétés de personnes sont soumises à un autre régime que les sociétés de capitaux, à savoir celui de l'impôt sur le revenu des particuliers. La majorité des sociétés de personnes est constituée d'entreprises individuelles ; celles-ci représentent $83 \%$ de toutes les sociétés recensées en Allemagne (IW Köln, 2008). S'y ajoutent les sociétés en nom collectif (offene Handelsgesellschaft, $O H G$ ), les sociétés de droit civil (Gesellschaft bürgerlichen Rechts, GBR) et les sociétés en commandite (Kommanditgesellschaft, KG). Un régime fiscal foncièrement différent s'applique aux sociétés de capitaux, c'est-à-dire aux sociétés par actions (Aktiengesellschaft, AG) et aux SARL (Gesellschaft mit beschränkter Haftung, GmbH).

Tableau 1 : Les sociétés de personnes prédominent dans le Mittelstand

\begin{tabular}{|c|c|c|c|c|}
\hline & \multicolumn{4}{|c|}{ Part des entreprises par catégorie de $C A^{*}$ ) } \\
\hline & faible & moyen & fort & Total \\
\hline & \multicolumn{4}{|c|}{ Part des entreprises ayant le statut de... } \\
\hline Nombre d'entreprises (en milliers) & 2514 & 514 & 9 & 3037 \\
\hline Sociétés de capitaux, dont... & $10,2 \%$ & $38,9 \%$ & $48,8 \%$ & $15,1 \%$ \\
\hline AG et KG a.A. & $0,1 \%$ & $0,8 \%$ & $8,7 \%$ & $0,2 \%$ \\
\hline GmbH & $10,1 \%$ & $38,1 \%$ & $40,1 \%$ & $14,9 \%$ \\
\hline Sociétés de personnes, dont... & $88,0 \%$ & $58,1 \%$ & $42,1 \%$ & $82,8 \%$ \\
\hline KG et GmbH \& Co. KG & $2,0 \%$ & $13,4 \%$ & $37,5 \%$ & $4,0 \%$ \\
\hline OHG et GBR & $8,4 \%$ & $9,7 \%$ & $3,2 \%$ & $8,6 \%$ \\
\hline Entreprises individuelles & $77,6 \%$ & $35,0 \%$ & $1,4 \%$ & $70,2 \%$ \\
\hline Autres (par ex. coopératives) & $1,8 \%$ & $3,1 \%$ & $9,2 \%$ & $2,1 \%$ \\
\hline
\end{tabular}

Source des données : Destatis, statistiques fiscales (taxe sur le CA) ; état : 2005. *) Faible CA : $\leq 500000 €$; CA moyen : de $500000 €$ à 50 millions $€$; fort $\mathrm{CA}:>50$ millions $€$

Le tableau ci-dessus dresse un aperçu de la répartition par statut juridique des entreprises allemandes dans les statistiques sur le chiffre d'affaires. Ces données ne tiennent donc pas compte du demi-million environ de micro-entreprises et de travailleurs indépendants (avocats, médecins, architectes, etc.) qui ne sont pas soumis à la taxe sur le CA, mais à l'IR comme toute société de personnes.

\section{La problématique particulière de la taxe professionnelle communale}

Un impôt Dans le cadre de la réforme s'est posé le problème particulier de la taxe professionnelle - littéralement : " taxe sur les activités industrielles et commerciales » (Gewerbesteuer) -, un impôt revenant en propre aux communes, et qui n'a pas d'équivalent dans la plupart des autres pays. Tout plaidait pour supprimer cette taxe communale sur les bénéfices ou pour l'intégrer sous une forme ou une autre dans l'IS ou l'IR, mais les communes se sont opposées à la suppression de cette taxe dont chacune d'entre elles fixe le montant en pleine autonomie.

Le taux de prélèvement est déterminé par les communes en prévision de leurs besoins budgétaires, mais aussi en fonction de la profitabilité des entreprises sises sur leur territoire. En 2004 a été créé un taux de prélèvement plancher de 
$200 \%$ pour réduire la concurrence fiscale émanant des petites communes. Car les grandes communes et les grandes villes pratiquent généralement des taux supérieurs à $400 \%$.

Le barème de la Gewerbesteuer se calcule de la manière suivante :

taux de prélèvement $x$ indice de la taxe professionnelle /100 = barème de la taxe professionnelle (en \%).

Comme dans les communes de taille moyenne, le taux de prélèvement et de $432 \%$, qu'il est multiplié par 3,5 puis divisé par 100 , ce barème s'élève généralement à $15,1 \%$.

L'indice de la taxe professionnelle - identique dans toutes les communes - a, quant à lui, été abaissé de $5 \%$ à $3,5 \%$, mais avec pour contrepartie (contrefinancement) un élargissement de la base de calcul de l'assiette. Avant la réforme de 2008, le calcul des bénéfices ainsi imposés intégrait $50 \%$ de tous les intérêts versés pour tout crédit courant souscrit pour une période supérieure à un an. Cette disposition a été supprimée en 2008. Depuis, entrent dans le calcul de l'assiette de la taxe professionnelle $25 \%$ de tous les intérêts payés comme de la part présumée du financement dans les dépenses pour baux et loyers, ainsi que les contrats de leasing. Dans le cas des biens mobiliers, cette part de financement et d'intérêts est estimée à $20 \%$; dans celui des biens immobiliers, à $75 \%$ (IW Köln, 2008). La charge fiscale des entreprises dépend donc très largement de leur situation individuelle (endettement, recours aux baux ou aux contrats de leasing).

La réforme a parallèlement élargi les possibilités offertes aux sociétés de personnes de déduire la taxe professionnelle de l'IR. En effet, comme seuls les revenus issus d'une activité industrielle et commerciale sont soumis à la taxe professionnelle, les sociétés de personnes subiraient, sans cette mesure compensatoire, une charge fiscale nettement plus lourde et risquant de grever leurs investissements. Les sociétés de personnes sont donc exemptées de la taxe professionnelle en dessous d'un seuil de $400 \%$ du taux de l'impôt prélevé. Or au-delà, c'est-à-dire dans les grandes communes et les villes, les possibilités de défalquer la taxe professionnelle de l'IR sont réduites à la portion congrue, à quoi s'ajoutent les effets négatifs d'une assiette de la Gewerbesteuer à la base de calcul désormais élargie.

\section{Une autre approche de la fiscalité pour les sociétés de personnes}

La réforme a également modifié en profondeur la logique de la fiscalité frappant les sociétés de personnes, étant donné qu'elle a introduit une distinction, inconnue jusque là du code allemand des impôts, entre les régimes applicables aux bénéfices distribués ou non (Brügelmann, 2009). Les bénéfices qui ne quittent pas l'entreprise connaissent désormais un régime plus favorable. Jusqu'en 2007 en effet, les sociétés de personnes étaient soumises exclusivement à l'IR, et sans aucune distinction entre le revenu de la société et celui de ses propriétaires. Les parts de bénéfices étaient attribuées directement à un sociétaire et imposées selon son barème individuel d'IR - quel que soit l'usage fait de ces bénéfices.

Depuis la réforme de 2008 , dans le cas des sociétés de personnes, les bénéfices thésaurisés connaissent donc un traitement plus favorable : ils ne sont plus imposés que selon un barème réduit de $28,25 \%$. Mais si, au cours des années suivantes, une partie de ces bénéfices est distribuée, la part prélevée est frappée, à titre rétroactif, d'un barème supérieur. Cela ouvre aux sociétés de personnes la possibilité de différencier les choix fiscaux pour l'entreprise, à l'image des sociétés de capitaux. Autrement dit: le sociétaire n'est fiscalement assimilé à un actionnaire que lorsque le bénéfice quitte l'entreprise.

Mais à la différence des sociétés de capitaux, les barèmes applicables ont en partie été augmentés dans le cas des sociétés de personnes. Car depuis le début de 2009, le barème de l'IR sur les tranches de revenus supérieures (45\%), surnommé " impôt sur les riches » («Reichensteuer »), et qui s'applique depuis 2007 aux revenus annuels de plus de $250000 €$ des particuliers, frappe aussi les revenus d'activités industrielles et commerciales. Mais de ce fait, le barème plafond est supérieur de 3 points de pourcentage à son niveau d'avant la réforme ; et si on y inclut la taxe de solidarité pour la reconstruction de l'économie est-allemande (Solidaritäts-
Un indice abaissé, mais une assiette élargie

Une plus forte déductibilité de I'IR

Distinction entre bénéfices distribués ou non

Dans ce dernier cas, un régime plus favorable

Les sociétés de personnes paient " l'impôt sur les riches » 
Le nouvel impôt libératoire pour les bénéfices distribués...

... n'apporte guère d'améliorations

Réduction à $31 \%$ pour les sociétés de capitaux

Objectif impossible à atteindre pour les sociétés de personnes

Leur charge fiscale peut aller jusqu'à plus de $48 \%$ zuschlag), il s'élève même aujourd'hui à 47,5 \%. Et même si la réduction de l'impôt prélevé sur les bénéfices non distribués abaisse la charge fiscale des sociétés de personnes à $30 \%$ environ, cette baisse reste purement théorique, la charge réelle étant nettement supérieure.

En ce qui concerne l'imposition rétroactive des bénéfices distribués à une date ultérieure, elle s'effectue dans le cadre du nouvel impôt libératoire (Abgeltungssteuer), créé lui aussi en 2009 dans le cadre de la réforme de la fiscalité des entreprises. Tous les revenus de capitaux privés - dividendes, intérêts, ainsi que les bénéfices distribués par les sociétés de personnes qui les avaient au préalable thésaurisés ou investis et qui étaient de ce fait imposés à un taux réduit - sont depuis le début de l'année imposés à un taux unique de $25 \%$. Comme dans ce cas aussi, la taxe de solidarité de $5,5 \%$ est prélevée sur le montant imposable dû, le taux réel s'appliquant alors s'élève à $26,4 \%$.

L'objectif de cet impôt libératoire est double. Il vise d'une part à éviter les déséquilibres entre la charge fiscale pesant sur les bénéfices des entreprises et les autres catégories de revenus ; d'autre part, il cherche à réduire les incitations à la fraude fiscale. II n'en reste pas moins que, pour une société de personnes, la combinaison entre le régime avantageux si les bénéfices ne sont pas distribués et l'impôt libératoire reste défavorable en comparaison de I'IR prélevé dans le cas de bénéfices directement distribués. Et même pour les sociétés de capitaux, cet impôt libératoire n'apporte aucune amélioration, puisqu'en contrepartie de son introduction a été supprimé le régime spécifique aux dividendes, qu'avait introduit la réforme fiscale de 2001 [voir REA 54/01; IB]. Pour éviter une double imposition des bénéfices - une fois que la société a payé l'IS et la taxe communale, l'IR (barème maximal) aurait en plus été prélevé sur le restant lors de la distribution de dividendes - la disposition aujourd'hui supprimée ne rendait imposable que la moitié de ces revenus (Halbeinkünfteverfahren). De facto, elle se traduisait par une division par deux de l'impôt sur le revenu des dividendes, ce qui avait abaissé son taux à 22,5\%. Depuis la réforme de 2008/09 et l'introduction de la "Reichensteuer ", ce taux a augmenté de 4 points.

\section{La charge fiscale après la réforme}

Le gouvernement a raté de peu son objectif d'abaisser à $30 \%$ la charge fiscale globale pesant sur les sociétés de capitaux : avec la réforme, elle est tombée de $39,5 \%$ à $31 \%$. La taxe communale sur les bénéfices s'élève désormais à $15,1 \%$ en moyenne, et l'impôt sur les sociétés à $15,8 \%$ si on y inclut la taxe de solidarité. Ce calcul (Brügelmann, 2009) est établi sur la base d'un taux de prélèvement moyen de $432 \%$ de la taxe professionnelle dans les communes de plus de 50000 habitants, sachant que c'est dans cette catégorie que se concentrent les activités industrielles et commerciales. Le ministère fédéral des Finances prend, lui, pour référence un taux de prélèvement moyen de $400 \%$ seulement, ce qui l'amène à calculer un taux global d'imposition de 29,8 \%.

En comparaison, la charge pesant sur les sociétés de personnes est nettement supérieure. La loi leur offre désormais deux options d'imposition des bénéfices, selon qu'ils sont distribués ou non, avec la possibilité de les combiner. Le critère de comparaison le plus pertinent est le cas de leur non distribution, car c'est sur lui seul que porte la baisse des prélèvements. La charge fiscale se compose alors de la taxe communale, du barème réduit de l'IR après défalcation de la taxe communale, ainsi que de la taxe de solidarité. En théorie, après addition de ces composantes, le taux global s'élève à $31 \%$ comme pour les sociétés de capitaux.

Or en réalité, il est impossible à atteindre. En effet, puisqu'il est prélevé sur les comptes de la société, l'impôt payé sur les bénéfices non distribués est lui aussi imposé au titre de l'IR personnel du ou des propriétaires. Quelle que soit la raison pour laquelle ces bénéfices sont prélevés, dès qu'ils quittent l'entreprise, ils ne relèvent plus du régime du barème réduit, et sont donc imposables selon le barème normal de I'IR. II n'y a qu'une exception, concernant le prélèvement effectué au titre de l'impôt sur les successions. Au total donc, si on additionne l'imposition du prélèvement effectué sur les bénéfices, le barème plafond de I'IR après défalca- 
tion de la taxe communale (taux de prélèvement moyen de $432 \%$ ) et la taxe de solidarité, la charge fiscale pesant sur les sociétés de personnes peut aller jusqu'à 48,6 \%. Si, en y incluant les dividendes distribués, le revenu global n'atteint pas $250000 €$, la charge fiscale sera inférieure de 3 points de pourcentage. Si on considère que le montant total des impôts sur les bénéfices est prélevé sur ces derniers, alors la charge fiscale frappant les bénéfices thésaurisés s'élève à $37,6 \%$, soit 6,6 points de plus que pour les sociétés de capitaux.

Tableau 2 : Une charge fiscale supérieure pour les sociétés de personnes (taux de prélèvement sur les bénéfices, compte tenu de la taxe professionnelle, de l'IR/IS et de la taxe de solidarité)

\begin{tabular}{|c|c|c|c|}
\hline \multicolumn{4}{|c|}{ Sociétés de personnes } \\
\hline \multicolumn{2}{|c|}{ Bénéfices distribués } & \multicolumn{2}{|c|}{ Bénéfices thésaurisés } \\
\hline Distribution immédiate & Imposition a posteriori & Charge théorique & Charge réelle \\
\hline $48,6 \%$ & $49,1 \%$ & $31,0 \%$ & $37,6 \%$ \\
\hline \multicolumn{4}{|c|}{ Sociétés de capitaux } \\
\hline \multicolumn{2}{|c|}{ Bénéfices distribués } & \multicolumn{2}{|c|}{ Bénéfices thésaurisés } \\
\hline \multicolumn{2}{|c|}{$49,2 \%$} & \multicolumn{2}{|c|}{$31,0 \%$} \\
\hline
\end{tabular}

Source : IW Köln, 2008 ; Brügelmann, 2009. NB : Sur la base d'un taux de prélèvement de la taxe professionnelle de $432 \%$, d'un barème de I'IR de $45 \%$ (de $47,5 \%$ compte tenu de la taxe de solidarité), et d'un taux de $25 \%$ de l'impôt libératoire (de $26,4 \%$ compte tenu de la taxe de solidarité). Taux en vigueur depuis le 01-01-2009.

Or les bénéfices de l'entreprise doivent aussi assurer la subsistance du patronpropriétaire. Comme la part des bénéfices thésaurisés ou réinvestis bénéficiant du barème réduit diminue proportionnellement à la hausse de la part des bénéfices distribués, la charge fiscale pour l'entrepreneur s'accroît en conséquence. Depuis le début de 2009 , celle-ci est de $48,6 \%$ lorsque les bénéfices sont distribués en totalité. Mais comme la taxe professionnelle n'est défalquée en totalité que lorsque le taux de prélèvement de cette dernière est inférieur à $400 \%$, le barème plafond pour les revenus des activités industrielles et commerciales est en réalité supérieur d'un point de pourcentage environ au barème plafond de l'IR.

Un taux d'imposition de $37,6 \%$ en cas de thésaurisation maximale des bénéfices est donc foncièrement irréaliste dans le cas le plus courant en Allemagne, où l'entreprise est managée par son propriétaire qui en tire sa subsistance. Dans la pratique, en effet, la charge fiscale dépasse pratiquement toujours les $40 \%$. Certes, les petites entreprises générant des bénéfices plus faibles subissent des charges moindres, puisque, du fait de la progressivité de l'IR, l'entrepreneur voit reculer son taux marginal d'imposition. Mais dans le cas de ces petites entreprises, la part des bénéfices prélevés pour assurer la subsistance de leur patron est forcément plus importante proportionnellement, ce qui a pour effet d'accroître en retour la charge fiscale qui pèse sur elles.

\section{Les sociétés de personnes, défavorisées en comparaison internationale}

L'Allemagne n'est pas la seule à chercher à réduire le poids des prélèvements fiscaux pesant sur ses entreprises. Entre 2007 et 2008, neuf pays industrialisés ont abaissé leurs taux d'imposition pour les sociétés de capitaux. Les baisses les plus importantes - de respectivement 9,75 et 8,5 points de pourcentage - ont été réalisées en Italie et en Allemagne. Dans ces deux pays, c'est surtout la taxe professionnelle qui a été réduite. Un seul pays se distingue : le Japon, où le poids global des prélèvements a légèrement augmenté.

Bien qu'elle ait été réduite de 8,5 points en Allemagne, la charge fiscale des sociétés de capitaux (31\%) reste relativement élevée en comparaison internationale. Quant à celle pesant sur les sociétés de personnes qui ne distribuent pas leurs bénéfices $(37,6 \%)$, elle figure parmi les plus élevées. Si l'objectif des $30 \%$ pour les sociétés de capitaux n'a pas été atteint, la réforme de la fiscalité des sociétés de personnes, quant à elle, s'assimile pratiquement à un échec. Cela est d'autant plus vrai que le régime plus favorable, tel qu'il s'applique actuellement aux bénéfices thésaurisés ou investis, n'est non seulement guère favorable en réalité, mais mène au contraire dans un certain nombre de cas à une hausse de la charge fiscale.
Imposition des revenus du patron : une prise en considération problématique

La charge fiscale des sociétés de capitaux reste élevée en comparaison internationale 
Tableau 3 : Les sociétés de personnes allemandes sont défavorisées en comparaison internationale (taux de l'IS dans les pays industrialisés)

\begin{tabular}{|c|c|c|c|}
\hline & 2008 & 2007 & Evol. (en points de \%) \\
\hline USA $^{\text {a) }}$ & $45,4 \%$ & $45,6 \%$ & $-0,2$ \\
\hline Japon & $42,3 \%$ & $40,7 \%$ & $+1,7$ \\
\hline RFA - sociétés de personnes $^{b)}$ (bénéfices thésaurisés) ${ }^{c)}$ & $37,6 \%$ & $46,3 \%$ & $-8,7$ \\
\hline France & $34,4 \%$ & $34,4 \%$ & 0,0 \\
\hline Belgique & $34,0 \%$ & $34,0 \%$ & 0,0 \\
\hline Canada ${ }^{\text {d) }}$ & $33,5 \%$ & $36,1 \%$ & $-2,6$ \\
\hline RFA - sociétés de capitaux ${ }^{\text {b) }}$ & $31,0 \%$ & $39,5 \%$ & $-8,5$ \\
\hline Espagne & $30,0 \%$ & $32,5 \%$ & $-2,5$ \\
\hline Luxembourg & $29,6 \%$ & $29,6 \%$ & 0,0 \\
\hline Suède & $28,0 \%$ & $28,0 \%$ & 0,0 \\
\hline Royaume Uni & $28,0 \%$ & $30,0 \%$ & $-2,0$ \\
\hline Italie & $27,5 \%$ & $37,3 \%$ & $-9,8$ \\
\hline Pays Bas & $25,5 \%$ & $25,5 \%$ & 0,0 \\
\hline Autriche & $25,0 \%$ & $25,0 \%$ & 0,0 \\
\hline Danemark & $25,0 \%$ & $28,0 \%$ & $-3,0$ \\
\hline Hongrie & $21,3 \%$ & $21,3 \%$ & 0,0 \\
\hline République Tchèque & $21,0 \%$ & $24,0 \%$ & $-3,0$ \\
\hline Suisse ${ }^{e)}$ & $20,7 \%$ & $24,2 \%$ & $-3,6$ \\
\hline Pologne & $19,0 \%$ & $19,0 \%$ & 0,0 \\
\hline Irlande & $12,5 \%$ & $12,5 \%$ & 0,0 \\
\hline
\end{tabular}

Source: Brügelmann, 2009. a) New York ; b) pour un taux de prélèvement de la taxe professionnelle de $433 \%$, y inclus l'ISF ("Reichensteuer "), étendu en 2009 aux bénéfices des entreprises ; c) pas de régime spécifique favorable pour les bénéfices thésaurisés avant 2008 ; d) Ontario ; e) Zurich.

Et si, sur la base des barèmes retenus dans le cadre de la réforme de la fiscalité des entreprises, on voulait obtenir pour les sociétés de personnes thésaurisant ou réinvestissant leurs bénéfices une charge fiscale comparable à celle des sociétés de capitaux, il conviendrait d'abaisser le barème de l'impôt sur le revenu à environ $21 \%$. L'alternative consisterait à ne plus soumettre à l'IR les bénéfices non distribués après que l'impôt sur les bénéfices a été prélevé.

\section{Les effets pervers de la réforme de l'impôt sur les successions}

Objectif : alléger la charge pesant sur les PME..

... si le successeur préserve patrimoine et salariés

Des conditions peu réalistes
Parmi les impôts qui pèsent le plus lourd sur les sociétés de personnes figure, outre celui sur les bénéfices, l'impôt sur les successions. Ce dernier revient en propre aux Länder, et son encours s'élève à environ 4 milliards $€$ par an. La réforme adoptée après plusieurs années de discussion par le gouvernement de Grande coalition (Bundesregierung, 2008 ; Pauli/Maßbaum, 2009), et entrée en vigueur au $1^{\mathrm{er}}$ janvier 2009 avec un an de retard, vise expressément à alléger la charge fiscale pesant sur le Mittelstand.

Le modèle retenu est le suivant : le montant imposable dû est réduit d'année en année si l'héritier reprend l'entreprise. Et celui-ci bénéficie d'un abattement de $85 \%$ s'il conserve le patrimoine de l'entreprise au-delà de 7 ans et si, durant ces 7 ans, il ne procède pas à des licenciements importants, c'est-à-dire faisant passer en dessous du seuil de $650 \%$ de son niveau initial la masse salariale cumulée. Quant aux $15 \%$ restants de la valeur de l'entreprise, ils bénéficient eux aussi d'un abattement, plafonné à $150000 €$ (Brügelmann/ Fuest, 2008). Si, durant cette période septennale, la masse salariale tombe en dessous du seuil, l'avantage fiscal est réduit en proportion. Par ailleurs, la distinction initialement prévue dans le traitement fiscal du patrimoine nécessaire à la marche de l'entreprise ou non n'a pas été retenue dans la loi de réforme lorsque la valeur d'exploitation non indispensable à l'activité reste inférieure à la moitié du patrimoine total de l'entreprise transmise au successeur (Lüdicke/Fürwentsches, 2009).

Comme l'estimation des biens transmis devait s'effectuer en fonction de leur valeur vénale, conformément au jugement rendu par le Tribunal constitutionnel fédéral le 7 novembre 2006 (1 BvL 10/02), ne pas adopter un tel abattement aurait considérablement accru la charge fiscale sur les PME et aurait mis en danger un grand nombre de successions. Toutefois, le seuil de $650 \%$ de la masse salariale (soit $93 \%$ par an) amène une hausse de l'impôt sur les successions pour les entreprises qui connaissent des difficultés économiques et sont de ce fait contraintes de 
réduire leurs effectifs. Le nouveau régime de l'impôt sur les successions pourrait donc amplifier les effets de la crise actuelle. En effet, en cas de compression d'effectifs incontournable du fait de la chute des ventes, une rectification fiscale est inévitable ; et celle-ci peut entraîner l'insolvabilité de l'entreprise.

Par ailleurs, les experts comme les entreprises concernées critiquent la complexité du nouveau code fiscal. L'estimation de la valeur du patrimoine nécessaire à la marche de l'entreprise comme de celle du patrimoine nécessaire à sa gestion implique la prise en considération d'une telle multitude de détails que les litiges entre administrations fiscales et héritiers sont littéralement programmés. S'ajoute à cela une disposition optionnelle qui ouvre droit à un abattement de $100 \%$ si l'activité de l'entreprise est maintenue pendant 10 ans par l'héritier et si la masse salariale est préservée dans sa totalité. Or cette option, qui ne peut être choisie qu'au moment de la succession, ne peut plus être annulée ensuite ; elle implique donc une estimation prospective de la marche des affaires à l'échéance de 7 ou 10 ans, ce qui équivaut à prendre un billet de loterie.

\section{Un contre-financement remis en question par la crise économique}

Pour limiter la baisse de l'encours fiscal induite par la réforme, la base de calcul de l'assiette et des barèmes a été élargie. Le gouvernement fédéral a ainsi rendu plus restrictives pour toutes les entreprises les modalités de présentation des comptes. Les principales modifications apportées sont la suppression de la dégressivité des amortissements sur les biens meubles investis et la suppression de l'imputation des charges dans l'assiette de la taxe professionnelle. Cela étant, dans le cadre du deuxième programme conjoncturel adopté en réaction à la crise économique mondiale, la disposition supprimant la dégressivité des amortissements a été assouplie temporairement : en 2009 et 2010, la dégressivité est appliquée au taux maximal de $25 \%$ ou bien au plus à deux fois et demi de celui de l'amortissement linéaire.

Pour contrefinancer la réforme a été par ailleurs introduite une limite d'intérêts (Zinsschranke) qui limite la déductibilité des coûts d'intérêts. Depuis 2008, les intérêts payés ne peuvent plus être déduits des bénéfices imposables si, après déduction du produit de l'intérêt, leur montant s'élève à plus de $30 \%$ de l'excédent avant intérêts, impôts et amortissements. Mais il existe une franchise de 1 million $€$. Cela signifie que les frais financiers nets restent déductibles, à condition qu'ils soient inférieurs à 1 million $€$. Cette limite d'intérêts ne concerne certes que les entreprises affiliées à un groupe, mais ce cas se présente dès qu'une société a des filiales à l'étranger et qu'elle peut présenter un bilan consolidé de groupe.

Du fait de ces deux restrictions, la limite d'intérêts, destinée aux sociétés de capitaux, s'applique également aux grandes entreprises familiales, mais non à la majorité du Mittelstand. Selon les estimations du ministère fédéral des Finances, quelque 300 entreprises seulement sont concernées - mais celles-ci avaient été effectuées avant la crise économique. Dans une étude réalisée en 2009 (BDI, 2009), la Fédération de l'Industrie estime ce nombre à 1500 (sur 70000 entreprises considérées). Du fait de la crise, le nombre d'entreprises tombant sous l'application de cette disposition pourrait nettement augmenter encore, étant donné que si le montant des intérêts qu'elles payent reste constant, il risque de dépasser de plus en plus souvent le seuil des $30 \%$ de l'excédent. Autrement dit, en tendance, la limite d'intérêts amplifie les effets de la crise. Conscient de ces risques, le gouvernement fédéral vient de décider à la fin mai d'élever la franchise de la limite d'intérêts à 3 millions $€$ pour les années 2009/10. Actuellement, la question de la limite d'intérêts ne concerne donc plus le Mittelstand.

En outre, le ministère fédéral des Finances a élargi l'assiette de la taxe professionnelle et introduit une taxe sur l'externalisation d'activités. Ces mesures sont censées générer une hausse des recettes de 3,8 milliards $€$, mais leur mise en œuvre dépend de la situation particulière de chaque entreprise. Au total, mesures de contre-financement incluses, le gouvernement fédéral estimait à 5 milliards $€$ la réduction de la charge fiscale pesant sur les entreprises. Mais comme les recettes issues de la fiscalité des entreprises s'étaient considérablement accrues jusqu'au milieu de 2008 du fait du boom des activités, avant de chuter spectacu-
Extrême complexité de l'estimation du patrimoine

Création d'une limite d'intérêts...

... mais elle risque d'amplifier les effets de la crise

Il est actuellement impossible d'évaluer l'impact de la réforme 
lairement sous l'effet de la crise, il est actuellement impossible d'évaluer sérieusement l'impact de la réforme de la fiscalité des entreprises.

LA RÉFORME A CERTES PERMIS À L'ALLEMAGNE DE REMONTER dans la moyenne supérieure des palmarès internationaux de la charge fiscale pesant sur les sociétés de capitaux (31\%), mais elle reste perfectible, surtout en ce qui concerne les sociétés de personnes, qui appartiennent majoritairement au Mittelstand. Ces PME sont soumises à I'IR, ce qui porte leur charge fiscale à $48,6 \%$ si on y inclut la «Reichensteuer » et la taxe communale. Certes, lorsque les bénéfices et autres revenus du patron-propriétaire sont en deçà de $250000 €$, ce taux est inférieur de 3 points. II n'en reste pas moins que dans presque tous les cas, le taux d'imposition des bénéfices distribués dépasse nettement les $40 \%$ pour les PME.

La réforme a aussi créé un régime plus avantageux pour les bénéfices non distribués. Mais vraisemblablement mû par l'intention de réduire le manque à gagner, le gouvernement fédéral a introduit une disposition selon laquelle le paiement de l'impôt afférent est considéré en droit fiscal comme une distribution des bénéfices, celle-ci étant imposable au titre de l'impôt sur le revenu privé du propriétaire-patron de l'entreprise. De ce fait, même en cas de thésaurisation du total des bénéfices, la charge fiscale pour les entrepreneurs soumis à l'ISF s'élève à plus de $37 \%$. Pour rétablir l'équité de traitement fiscal avec les sociétés de capitaux, il conviendrait de ne plus taxer au titre de l'IR du propriétaire le paiement des impôts de la société.

En ce qui concerne la taxe professionnelle communale, le projet de réduire le poids des prélèvements fiscaux pour les sociétés de personnes grâce à la défalcation de la taxe communale payée de l'impôt sur le revenu dû, a certes en grande partie abouti. Mais l'opportunité qui se présentait de simplifier le code fiscal grâce à la suppression de la taxe communale n'a pas été saisie. Pourtant, la solution d'intégrer cette taxe dans l'IS et l'IR est prônée depuis longtemps (cf. $\mathrm{BDI} / \mathrm{VCI}, 2001)$. En effet, comme sa base de calcul diffère de celle des autres impôts sur les bénéfices, un prélèvement sur l'IR et l'IS dont le fruit serait attribué aux communes permettrait de maintenir à niveau constant l'encours de ces dernières tout en réduisant significativement la charge administrative pesant sur les entreprises. Or la mise en œuvre de tels modèles s'est heurtée à la farouche opposition des communes, désireuses de conserver à tout prix 'leur' impôt - alors même que son fruit est plus sensible aux effets d'une crise que ne le serait une quote-part prélevée sur l'IR. Une réforme véritablement de fond de la fiscalité des entreprises en appelle dès lors une autre en préalable : celle de la répartition des impôts entre Bund, Länder et communes.

Traduction : I. Bourgeois

\section{Indications bibliographiques}

- Bundesverband der DeUtSChen INDUSTRIE, BDI, Die Zinsschranke - eine ungewollte Verschärfung der Krise, Berlin, 2009, www.bdi.eu

- Bundesverband der Deutschen Industrie, BDi / Verband der Chemischen Industrie, VCI, Verfassungskonforme Reform der Gewerbesteuer - Konzept einer kommunalen Einkommens- und Gewinnsteuer, Francfort/Main, Berlin, 2001

- BRÜGelmanN R., « Die Unternehmenssteuerbelastung », in IW-Trends, 3/2009

- BRÜgelmann R., Fuest W., "Erbschaftsteuerreform - Eine halbherzige Lösung », in IW-Positionen, $\mathrm{n}^{\circ} 34,2008$

- BundesRegieRUNG, «Gesetz zur Reform des Erbschaftsteuer- und Bewertungsrechts - Erbschaftsteuerreformgesetz (ErbStRG) », 2008, Bundesratsdrucksache 888/08

- Institut der Deutschen Wirtschaft, IW (ed), "Mittelstand von A bis Z », IW Dossier, n 34 , 2008

- LÜDICKE J., FüRWENTSCHES A., «Das neue Erbschaftsteuerrecht », in Der Betrieb, vol. 01-02/ 2009

- PaUli R., Maßbaum M. (eds), Erbschaftsteuerreform 2009 - Beratungsschwerpunkte und Checklisten, Cologne, 2009. 